\title{
Position distinctiveness and successive discrimination learning*
}

\author{
DOUGLAS L. MEDIN. \\ Rockefeller University, New York, New York 10021
}

\begin{abstract}
This experiment tested the prediction that increased spatial separation of discriminanda would facilitate successive discrimination learning. Eight pigtailed monkeys were trained concurrently on two successive discrimination problems, one with a small and the other with a large separation of cues. Better performance was associated with the larger cue separation.
\end{abstract}

-Although it has not been overly popular, the successive discrimination paradigm is of appreciable value is assessing discrimination learning theories. For example, the failure of Spence's original theory (1936) to predict that the successive problem is soluable led Spiker $(1963,1970)$ to hypothesize that the strength of a habit accruing to a stimulus feature in one compound is diminished when that feature appears in another compound to the degree that the two compounds are dissimilar overall. Spiker's modification, known as the stimulus interaction hypothesis, greatly increased the power and range of applicability of Spence's theory and correctly predicted that successive discriminations were soluable.

Working from a quite different orientation, Medin (1973) proposed that the amount of information associated with a stimulus in a given context is reduced by changes either in the stimulus or in its context. Both Medin's theory and Spiker's theory predict that successive discrimination performance should be facilitated by distinctive positional cues. The present paper reports a test of this prediction.

The paradigm used is shown in Table 1. The four settings are presented concurrently, and the comparison of interest is performance on Pairs A and B having a small spatial separation of stimuli vs Pairs C and D having a larger spatial separation of stimuli. One might suspect that a between-Ss design is called for, but the predictions of the theories under consideration hold equally for concurrent presentations. Therefore, it is possible to take advantage of the greater power of a within-Ss design. To my knowledge, no one has studied the effects of stimulus separation on successive discrimination learning in animals. According to Spiker and Medin, greater stimulus separation should lead to better performance.

\section{METHOD}

\section{Subjects}

The Ss were eight jungle born pigtailed monkeys (Macaca nemestrina), 3 to 4 years old. All animals had received approximately 1 year of training on various simultaneous discrimination problems, but no animal had had experience with successive discriminations.

*This research was supported by U.S. Public Health Service Grant MH 25134-01.

\section{Apparatus}

A Wisconsin General Test Apparatus was used with a three-foodwell formboard painted red-orange. Two of the foodwells, spaced $12 \mathrm{~cm}$ apart, were on either side of the center of the foodtray, while the third foodwell was on the far right of the foodtray, spaced $18 \mathrm{~cm}$ from the nearest foodwell. Objects either covered the two center foodwells and were $12 \mathrm{~cm}$ apart or covered the two most distant foodwells and were $30 \mathrm{~cm}$ apart. The stimuli were four pairs of objects as distinctive as possible. They differed on many dimensions including color, form, and height. The stimuli were approximately equal in width $(4 \mathrm{~cm})$ to provide uniformity in the stimulus separations.

\section{Procedure}

All animals were given successive discrimination problem shown in Table 1. The objects were assigned to spatial separations and reward conditions in a random balanced manner. Training was given in 40 trials each day for 9 days. The four settings were concurrently presented in a random order with the constraint that each setting appear twice in a block of eight trials. A noncorrection procedure was employed, and each correct response was rewarded with a raisin. The intertrial interval was approximately $12 \mathrm{sec}$.

\section{RESULTS AND DISCUSSION}

Figure 1 shows performance on the four settings referred to in Table 1. Initially, monkeys avoided responding to the most distant foodwell (producing excessive errors on $\mathrm{C}$ pair), but this tendency disappeared quickly. By the end of training, all eight monkeys responded more accurately to the large stimulus separations (C and D) than to the small stimulus separations (A and B).

Statistical analysis supports these trends. The effects of stimulus separation $(\mathrm{F}=11.11, \mathrm{df}=1 / 7, \mathrm{p}<.05)$ and 3-day blocks of practice $(F=6.59, \mathrm{df}=2 / 14, \mathrm{p}<.01)$ were significant, while the Stimulus Separation by

Table 1

The Experimental Paradigm

\begin{tabular}{clll}
\hline Setting & & & \\
\hline 1 & A & A+ & \\
2 & B+ & B & \\
3 & C & & C+ \\
4 & D+ & & D \\
\hline
\end{tabular}

Note-A, B, $C$, and $D$ are four distinct pairs of stimuli. Stimuli appear in one of three spatial positions producing either small or large stimulus separations. The plus sign indicates the reward placements. 


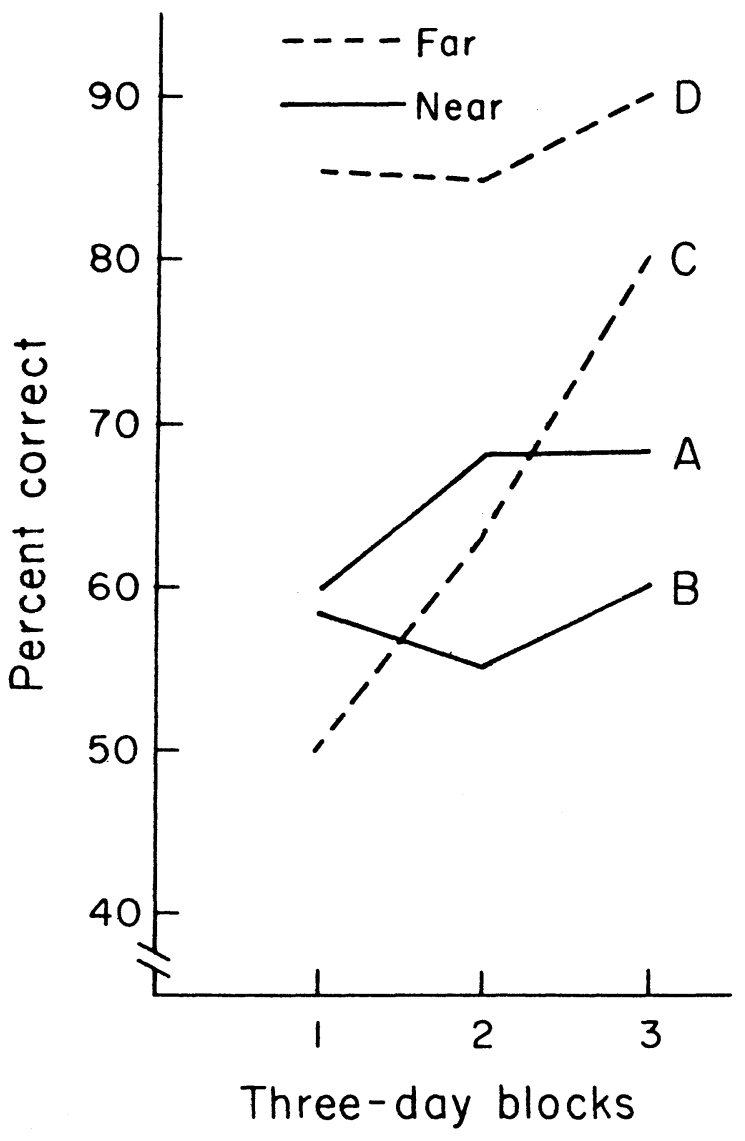

Fig. 1. Percentage of correct responses on each of the four settings of the successive problem as a function of 3-day blocks of practice.

Practice interaction approached significance $(F=3.59$, $\mathrm{df}=1 / 7, \mathrm{p} \cong .06$ ).
Stimulus separation did facilitate successive discrimination learning. According to the theories mentioned earlier, the primary basis for this effect is that increased distances between stimuli within a setting should decrease the generalization between these stimuli. This would facilitate the development of differences in habit strength or information associated with these stimuli which provides the basis for efficient discrimination performance.

Differences between Spiker's theory and Medin's theory are considered elsewhere (Medin, 1973; Flagg, 1974). The main assumption the theories have in common is that the features comprising a stimulus compound or complex are not assumed to be handled independently. This allows the theories to predict that successive discriminations are soluble and that position distinctiveness will facilitate this discrimination as was shown in the present experiment.

\section{REFERENCES}

Flagg, S. F. Learning of the insoluable conditional reaction problem by rhesus monkeys. Animal Learning \& Behavior, 1974 , in press.

Medin, D. L. Context in primate discrimination learning. Paper presented at Psychonomic Society meetings, St. Louis, November 1973.

Spence, K. W. The nature of discrimination learning in animals. Psychological Review, 1936, 43, 427-449.

Spiker, C. C. The hypothesis of stimulus interaction and an explanation of stimulus compounding. In L. P. Lipsitt and C. C. Spiker (Eds.), Advances in child development and behavior. Vol. 1. New York: Academic Press, 1963.

Spiker, C. C. An extension of Hull-Spence discrimination learning theory. Psychological Review, 1970, 77, 496-515.

(Received for publication April 15, 1974.) 